\title{
Investigation of stopping criterion for OSEM algorithm with application to nuclear medicine
}

\author{
N.V. Denisova ${ }^{1}$, O. Krivorotko ${ }^{2,3}$ \\ ${ }^{1}$ Novosibirsk State University, Novosibirsk, Russia \\ ${ }^{2}$ Khristianovich Institute of Theoretical and Applied Mechanics, Novosibirsk, Russia \\ ${ }^{3}$ Institute of Computational Mathematics and Mathematical Geophysics SB RAS, Novosibirsk, Russia \\ *e-mail: lijiyu1234@yandex.ru
}

Key words: inverse problem, SPECT, PET, OSEM, optimization, regularization

Motivation and Aim: The OSEM (Ordered Subset Expectation Maximization) algorithm $[1,2]$ is studied in this work. A diagnostically acceptable image is obtained by interrupting (stopping) of the iterative process because the OSEM algorithm is developed on the basis of an unregularized approach. In fact, the interrupt is a "rough regularization". The iteration number of the "stop of the algorithm" is determined in most cases empirically in preliminary studies and recorded in the patient examination protocol for a particular type of installation. The doctor must follow the appointed protocol. However, patients differ in their anatomical constitutions therefore the requirements of the protocol do not always correspond to the obtaining of the optimal image.

Methods and Algorithms: It was suggested to use the Pearson statistical criterion Chi-square as the stopping rule [3]. However, this proposal was not implemented on commercial installations. A theoretical analysis of regularization of OSEM based on stochastic properties of process and mathematical analysis of misfit function is carried out [4].

Results: In this work, studies of the OSEM image reconstruction algorithm are performed in the context of applications to positron emission tomography (PET) and single-photon emission computed tomography (SPECT)]. It is shown theoretically and in numerical simulation that if the source function is stochastic and includes regions with very different levels of statistics of emitted gamma quanta, the Pearson criterion gives incorrect values of "stopping". Our research has shown that the reason is that regions with different statistics behave differently in the iterative process and give different values for the stopping criterion.

Acknowledgements: Supported by the Russian Foundation for Basic Research (No. 1752-14004).

\section{References}

1. Shepp L.F., Vardi Y. (1982) Maximum Likelihood reconstruction for Emission Tomography IEEE Trans. Med. Imag. 1(2):113-121.

2. Hudson H., Larkin R.S. (1994) Accelerated Image Reconstruction Using Ordered Subsets of Projection Data IEEE Trans. Imag. Imag. 13(4):601-609.

3. Veclerov E., Llacer J. (1987) Stopping Rule for the MLE Algorithm Based on Statistical Hypothesis Testing IEEE Trans. Med. Imag. 6(4):313-319.

4. Kabanikhin S.I. (2008) Definitions and examples of inverse and ill-posed problem. Journal of Inverse and Ill-Posed Problems. 16(4):317-357. 